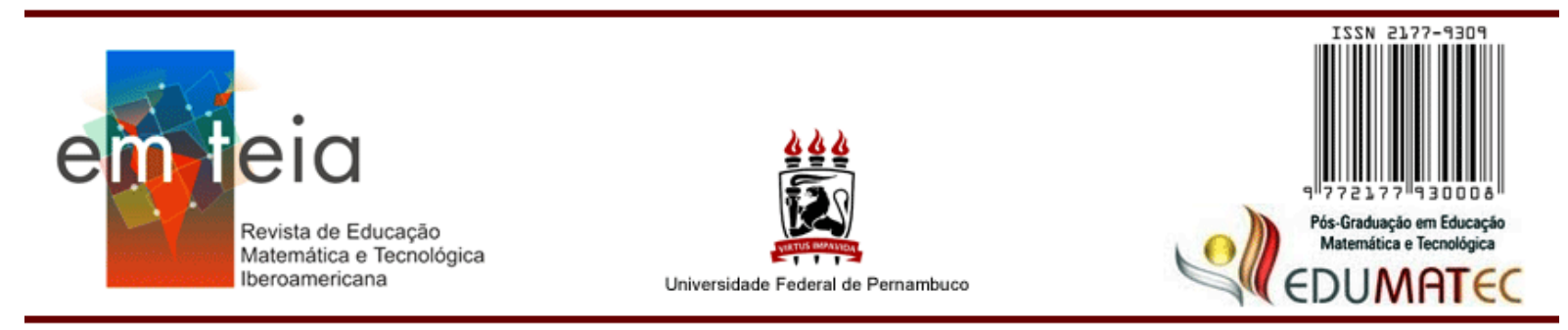

\title{
APREENSÕES OPERATÓRIAS EM REGISTROS FIGURAIS: um estudo com alunos de Licenciatura em Matemática
}

\section{OPERATIVES APPREHENSIONS IN FIGURAL RECORDS:}

\author{
a study with graduation students in Mathematics
}

\author{
Mariana Moran \\ Doutorado em Educação Matemática \\ Universidade Estadual do Paraná/Campo Mourão - Paraná - Brasil \\ marianamoranbar@gmail.com \\ Carla Larissa Halum Rodrigues \\ Graduação em Licenciatura em Matemática \\ Universidade Estadual do Paraná/Campo Mourão - Paraná - Brasil \\ carlalarissahalumrodrigues@hotmail.com
}

\section{Resumo}

Este trabalho investiga o uso dos Registros de Representação Semiótica na Geometria. Para isso, foi observada a influência dos Materiais Manipuláveis (MM), do Software GeoGebra (SG) e das Expressões Gráficas (EG) como registros figurais nas apreensões operatórias de figuras geométricas. As referidas apreensões foram estudadas com base na Teoria dos Registros de Representação Semiótica, de Raymond Duval, e dizem respeito às possíveis modificações que podem ser realizadas em registros figurais com o intuito de resolver um problema de geometria. Para esta investigação, realizamos a aplicação de uma atividade com 12 licenciandos do $3^{\circ}$ e $4^{\circ}$ anos de Matemática de uma Universidade ao norte do Estado do Paraná. A atividade foi aplicada individualmente e elaborada com o propósito de pesquisar os indícios de apreensões operatórias que o contato com os registros figurais na forma de MM, SG e EG proporciona. A análise dos dados foi realizada com base nos objetivos propostos, nas resoluções escritas pelos alunos e áudios gravados durante a aplicação da atividade. Com esta pesquisa, constatamos que a diversidade de registros figurais contribui para a visualização de conceitos e propriedades dos objetos geométricos em questão, auxiliando na resolução do problema, proporcionando diferentes operações e completando um registro figural ao outro.

Palavras-Chave: Geometria. Representação semiótica. Registros figurais. Apreensão operatória.

\begin{abstract}
This work investigates the use of Registers of Semiotic Representation in Geometry. For this, we have observed the influence of Manipulatives (MM), GeoGebra software (SG) and Graphic Expressions (EG) as figural records in operative apprehensions of geometric figures. These apprehensions were studied based on the Theory of Registers of Semiotic Representations, by Raymond Duval, and deal with possible modifications that can be performed in figural records in order to solve a geometry
\end{abstract}


problem. For this research, we conducted the implementation of an activity with 12 undergraduates from the 3rd and 4th grades of Mathematics, in a University of North Paraná. The activity was individually applied and developed in order to search for evidence of operative apprehensions provided by the contact with the figural records as MM, SG and EG. Data analysis was based on the proposed objectives, resolutions written by the students and audios recorded during the activity application. With this research, we found that the diversity of figural records contributes to the visualization of concepts and properties of the geometric objects in question, assisting in the resolution of the problem by providing different operations and completing one figural record to another.

Keywords: Geometry. Semiotic representation. Figural records. Operative apprehension.

\section{Introdução}

A presente proposta consiste em investigar as apreensões operatórias nos registros figurais na forma de Materiais Manipuláveis, do Software GeoGebra e das Expressões Gráficas, com foco na exploração heurística das figuras e na congruência existente entre as hipóteses de um enunciado e os tratamentos figurais a serem realizados durante a resolução de um problema de geometria.

As apreensões operatórias foram estudadas com base na Teoria dos Registros de Representação Semiótica de Raymond Duval e dizem respeito às modificações figurais realizadas pelos sujeitos no momento da atividade quando eles têm contato com um registro figural como representação para um objeto geométrico.

A importância em investigar as apreensões operatórias dos registros figurais é justificada pelo fato de que no trabalho com a representação geométrica a análise de suas figuras e os tratamentos realizados são fundamentais para a construção do conhecimento dos conteúdos envolvidos.

Dessa forma, os resultados desta pesquisa são importantes principalmente porque constatou-se que dependendo do registro figural apresentado os alunos manifestam procedimentos de resoluções diferentes, e também para mostrar uma possibilidade de ensino de geometria a partir dos registros figurais.

Além disso, proporciona parte do conhecimento da Teoria dos Registros de Representação Semiótica de Duval e apresenta possíveis representações para uma mesma figura geométrica auxiliando professores no ensino da geometria e na formação inicial e continuada de professores de matemática.

Assim, norteamos a pesquisa pelo seguinte problema: como o Material Manipulável, o Software GeoGebra e a Expressão Gráfica influenciam nas apreensões operatórias de figuras em uma atividade de Geometria com alunos de Licenciatura em Matemática? 
Para encontrar a resposta deste problema tivemos como objetivo investigar a influência dos Materiais Manipuláveis, do Software GeoGebra e das Expressões Gráficas como registros figurais nas apreensões operatórias de figuras no estudo da Geometria com licenciandos de Matemática.

\section{Os registros de representação semiótica em geometria}

Em geometria, os registros figurais são compostos por figuras geométricas e utilizados para representar os entes matemáticos. Os registros figurais direcionam os alunos a terem outras interpretações durante a resolução de uma atividade de geometria.

Duval (2011) apresenta três características que atribuem aos registros das figuras um poder cognitivo particular: inicialmente, elas têm um valor intuitivo - a maneira de ver a figura sem precisar de explicações; em seguida, elas dão lugar ao reconhecimento de objetos como imagens desenhadas; e, por fim, podem ser "construídas instrumentalmente seja com régua, com o compasso ou com um software" (DUVAL, 2011, p. 84).

Neste trabalho, consideraremos como registros de representação semiótica em geometria os registros figurais representados por figuras geométricas por meio de Materiais Manipuláveis (MM), de construções figurais no Software GeoGebra (SG) e de Expressões Gráficas (EG). Essas três formas de representar as figuras possibilitam realizar operações.

Duval (2012b) afirma que um registro de representação semiótica deve permitir três atividades cognitivas fundamentais:

- A formação de uma representação identificável como uma representação de um registro dado: que deve respeitar regras de utilização, de identificação, de reconhecimento da representação e a possibilidade de sua utilização para tratamentos.

- O tratamento: é a transformação de uma representação no mesmo registro.

- A conversão: é a transformação de uma representação em outro registro, conservando a totalidade ou uma parte somente do conteúdo da representação inicial.

A forma de representação do objeto influencia diretamente na compreensão de seu conceito e nas propriedades tornando fundamental o tipo de representação exigida pela situação em que se trabalha, por isso a importância de trabalhar com o maior número possível de registros de representação de um mesmo objeto, até que haja um desvencilhamento do objeto à sua representação e a aprendizagem do conteúdo referente.

Pensando nas formas de representar figuras geométricas, é possível utilizar Materiais Manipuláveis para tais representações. Passos (2006, p. 78) afirma que "os materiais 
manipuláveis são caracterizados pelo envolvimento físico dos alunos numa situação de aprendizagem ativa". No presente artigo, entende-se por MM todo material útil ao ensino que pode ser manuseado, desde poliedros construídos a recortes em cartolina. Uma outra forma de representar as figuras, além dos MM, é por meio do Software GeoGebra. Os softwares pedagógicos podem fornecer “[...] suporte as concretizações e ações mentais do aluno; isto se materializa na representação dos objetos matemáticos na tela do computador e na possibilidade de manipular estes objetos via sua representação" (GRAVINA; SANTAROSA, 1998, p. 10).

Desse modo, opta-se como registro figural pelo software GeoGebra, porque ele pode ser utilizado para representar figuras geométricas e "utilizando software, confere às figuras uma confiabilidade e uma objetividade que permitem efetuar verificações e observações" (DUVAL, 2011, p. 84).

O software livre GeoGebra contém vários comandos e ferramentas que proporcionam outra maneira do sujeito pensar sobre o registro auxiliando de forma rápida na resolução de atividades de geometria, estatística, álgebra e de outros conteúdos.

Além do MM e do SG, outra forma muito utilizada para representar as figuras é por meio da Expressão Gráfica. Considera-se neste trabalho a Expressão Gráfica como imagens construídas no papel sulfite que formam figuras passíveis de comunicar uma ideia, um conceito. Para Góes (2012, p. 53), ela "pode auxiliar na solução de problemas, na transmissão de ideias, de concepções e de pontos de vista relacionados a tais conceitos". A Expressão Gráfica é o tipo de registro figural mais utilizado nas aulas de Geometria, pois ela compõe as figuras dos livros didáticos de Matemática; dessa forma, quando os alunos entram em contato com outros registros sentem dificuldades em identificar o objeto. Uma forma de sanar essas dificuldades é trabalhar com os diferentes registros de representações figurais nas aulas de geometria.

Nota-se que, em cada situação, seja com os Materiais Manipuláveis, Softwares de Geometria ou com as Expressões Gráficas, o sujeito por meio de seu conhecimento pode realizar operações na figura buscando chegar a uma resolução num contexto de uma atividade.

\section{Apreensão operatória de figuras}

O uso de uma figura para a resolução de um problema possibilita ao sujeito realizar interpretações. Para essas interpretações, Duval (2012a) distingue quatro maneiras de apreensões figurais: sequencial, perceptiva, operatória e discursiva. Este trabalho trata da 
apreensão operatória de figuras: ela permite ver a diversidade de subfiguras e possibilita a realização de tratamentos a fim de encontrar uma resolução para a atividade.

A respeito da apreensão operatória de figuras, Duval (2012a, p. 125) afirma que "é uma apreensão centrada nas modificações possíveis de uma figura e nas reorganizações possíveis destas modificações", de forma a contribuir para resolução de algum problema proposto de matemática. Estas modificações são subdivididas por Duval (2012a) do seguinte modo:

- modificação mereológica: dividir uma figura em várias subfiguras; incluir uma figura em outra figura a fim dela se tornar uma subfigura, ou seja, esse tipo de modificação ocorre em função da relação parte e todo;

- modificação ótica: aumentar, diminuir ou até mesmo deformar uma figura transformando-a em outra de modo que esta seja sua imagem;

- modificação posicional: deslocar, rotacionar, refletir a figura com relação ao campo de referência em que ela se encontra.

Neste trabalho, será abordada, principalmente, a modificação mereológica, pois ela permite a aplicação da operação de reconfiguração intermediária possibilitando a produtividade heurística da figura na resolução de problemas de geometria. Desse modo, o fracionamento que acontece na figura juntamente com sua reorganização é o que origina a operação de reconfiguração intermediária. Por exemplo: transformar um retângulo em um paralelogramo (DUVAL, 2005, p. 14), conforme a Figura 1:

Figura 1: Fracionamento da figura e reagrupamento das subfiguras

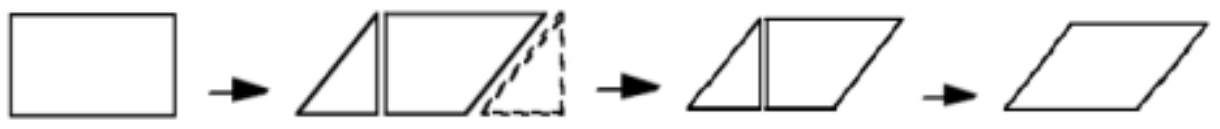

Fonte: Duval, 2005, p. 14.

Ao trabalhar com tais operações em registros figurais é possível, e muitas vezes necessário, associá-las a raciocínios dedutivos que podem ser expressos por meio de tratamentos matemáticos, ou seja, explorar uma figura de acordo com suas possibilidades heurísticas.

Nesse sentido, Duval (2012a, p. 125) aborda que "a produtividade heurística de uma figura em um problema de geometria, está ligada a existência da congruência entre uma destas operações e um dos tratamentos matemáticos possíveis para o problema proposto".

Em relação à produtividade heurística, acrescentam Flores e Moretti (2004, p. 8): 
A produtividade heurística de uma figura está ligada, de um certo modo na interação entre a figura e o texto, ou a descoberta na figura de um caminho para a solução do problema, o que implica aqui na descoberta de um modo de reconfiguração que esteja de acordo com o que é solicitado no problema.

A congruência entre as hipóteses dadas no enunciado e os tratamentos contribui para exploração heurística da figura.

Ao resolver o problema em diferentes registros o aluno deverá explorar heuristicamente a figura, realizar operações que poderão ser erradas ou certas, ou seja, realizar tratamentos que podem direcionar a um raciocínio dedutivo correto. Contudo, a maneira de interpretar o problema e a figura é de fundamental importância para a sua resolução correta e para aprendizagem da matemática.

\section{Metodologia e estratégias de ação}

A presente proposta caracteriza-se como uma pesquisa de abordagem qualitativa de caráter investigativo que tem por objetivo pesquisar: quais as influências das figuras representadas por meio do $\mathrm{MM}, \mathrm{SG}$ e EG no que diz respeito às apreensões operatórias em figuras geométricas com licenciandos de Matemática.

Para tanto, uma atividade foi aplicada individualmente com doze licenciandos do $3^{\circ} \mathrm{e}$ $4^{\circ}$ anos de Matemática de uma Universidade ao norte do Estado do Paraná, sendo 6 alunos do $3^{\circ}$ ano e 6 alunos do $4^{\circ}$ ano, que foram convidados e aceitaram participar da pesquisa. As turmas foram escolhidas pelo fato dos licenciandos em matemática já terem cursado a disciplina de geometria plana.

Antes da aplicação da atividade construímos os registros figurais na forma de Expressão Gráfica, Material Manipulável e Software GeoGebra e os doze alunos foram divididos em três grupos contendo quatro alunos em cada e para cada grupo foi apresentada uma sequência diferente dos registros figurais sendo: o Grupo 1 com a sequência $\{E G, S G$, $\mathrm{MM}$ \}; o Grupo 2 com a sequência $\{\mathrm{SG}, \mathrm{MM}, \mathrm{EG}\}$; e o Grupo 3 com a sequência $\{\mathrm{MM}, \mathrm{EG}$, SG $\}$.

Iniciamos a aplicação, disponibilizando ao participante um único problema com o enunciado digitado em uma folha com espaço para as resoluções e os materiais necessários para a resolução dos problemas, explicando que a atividade seria resolvida por meio de uma sequência de três registros figurais já definidos. Deste modo, cada licenciando tentava resolver a atividade por determinado registro e descrevia a sua resolução, em seguida tentava 
resolver a mesma atividade por meio de outro registro que lhe foi disponibilizado e descrevia a sua resolução, assim sucessivamente, percorrendo os três registros figurais.

Cabe ressaltar que independente de o aluno conseguir ou não resolver a atividade em determinado registro, o próximo registro figural foi apresentado de modo a explorar se o problema seria resolvido ou se seria resolvido com operações diferentes das efetuadas diante do registro anterior apresentado.

A coleta dos dados foi efetuada com base nas resoluções escritas dos estudantes e gravações de áudio das falas durante a aplicação. Para fazermos a análise, os alunos foram indicados por A1, A2, A3, e assim, sucessivamente, até A12 e foram consideradas as resoluções escritas e as transcrições de áudio de cada aluno.

As seguintes observações nortearam a análise dos dados quanto a identificar a apreensão operatória das figuras:

- se foram realizadas modificações (mereológicas, posicionais e óticas) e reorganizações com o intuito de resolver o problema. Se houve modificações, identificar o(s) tipo(s);

- se houve possibilidade de exploração heurística da figura (congruência entre os tratamentos e o raciocínio dedutivo);

- se o tipo de registro figural possibilitou congruência entre as hipóteses dadas no enunciado e os tratamentos.

\section{Atividade}

O problema de Euclides: mostrar a igualdade das áreas 1 e 2, qualquer que seja a posição do segmento $\overline{A B}$ (DUVAL, 1999, p. 157).

Figura 2: Imagens dos registros de representação

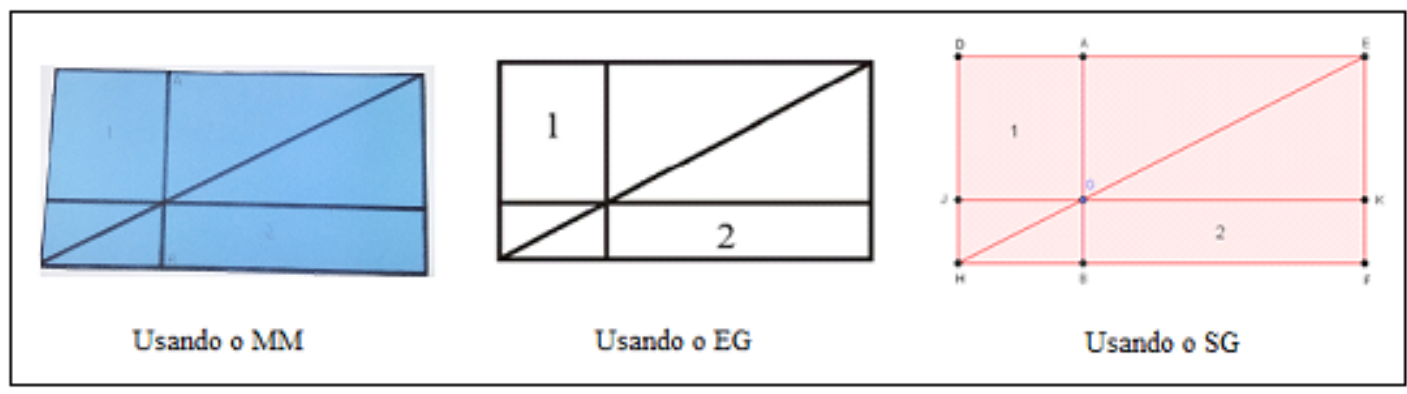

Fonte: Duval (2012b, p. 129) e autoras.

Essa atividade pode ser resolvida por uma modificação figural do tipo mereológica, fazendo-se uma operação de reconfiguração, que consiste no fracionamento da figura inicial em subfiguras e na modificação das subfiguras estabelecidas em uma figura. Nesse caso, pela 
supressão sucessiva de duas partes elementares iguais, que estão separadas pela diagonal do retângulo, conclui-se a igualdade das áreas 1 e 2, conforme a figura a seguir:

Figura 3: Primeira Solução da Atividade

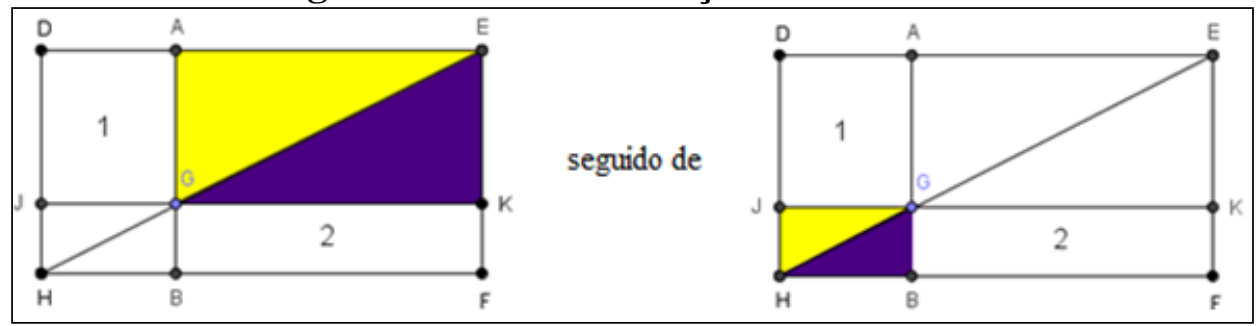

Fonte: Autoras

\section{Análise da atividade}

Com esta atividade analisamos as modificações operatórias realizadas na figura nos diferentes registos figurais: Materiais Manipuláveis (MM), Software GeoGebra (SG) e Expressão Gráfica (EG), ou seja, buscamos analisar se no momento de resolução da atividade ocorreram as seguintes modificações operatórias: a Modificação Mereológica, para isso foi necessário observar se as subfiguras que compõem a figura de partida ficam visíveis aos participantes, se ocorrem reconfigurações e se é estabelecida uma relação parte e todo nos registros disponíveis; a Modificação Ótica, para isso foi necessário observar se o registro figural apresentado permitiu que a figura de partida ou as subfiguras fossem aumentadas ou diminuídas de tamanho, transformando a figura de partida em outra de modo que esta seja sua imagem; a Modificação Posicional, para isso foi necessário observar se houve deslocamento, rotação e reflexão da figura com relação ao campo de referência em que ela se encontra.

Neste trabalho, também analisamos se houve congruência entre as hipóteses dadas no enunciado do problema e os tratamentos realizados nos diferentes tipos de registros figurais e será referido sobre a mobilização de outros registros realizada pelos alunos no momento da resolução da atividade.

As análises foram realizadas por grupos e a ordem dos registros figurais foi alternada em cada grupo de alunos, de modo a permitir que os dados para a análise fossem mais consistentes, porém relataremos nesse trabalho os resultados observados nos três grupos de modo concomitante.

\section{Resultados}

A Teoria dos Registros de Representação Semiótica em Geometria, mais especificamente o estudo das apreensões operatórias, foi essencial para direcionar as análises 
dos dados que consistiu em investigar a influência das figuras representadas por meio do Material Manipulável (MM), do Software GeoGebra (SG) e da Expressão Gráfica (EG) nas apreensões operatórias de figuras.

A análise da atividade proposta neste artigo evidencia a importância de utilizar diversos registros figurais, pois percebemos que para cada tipo de registro figural podem surgir modificações diferentes realizadas em prol de encontrar a solução para a atividade, como podemos observar pela fala do aluno A11:

A11: Se encontrar a distância entre os pontos, pode-se calcular as áreas e verificar se seriam iguais.

Pesquisadora: No $S G$ dá para calcular a área utilizando a ferramenta polígono.

A11: 3,33 agora vamos ver a área desse outro.

Pesquisadora: 3,33.

A11: Então é igual. Então porque quando recortei não deu?

Pesquisadora: Não sei.

A11: É que também não sei as opções que tem aqui (no $S G$ ). Eu acho que sempre vai ser igual mesmo, porque aqui está a diagonal né, e esse ponto aqui só anda na diagonal então essa área vai ser igual àquela mesmo.

Pesquisadora: Por quê?

A11: Porque aqui é a metade do retângulo que formam dois triângulos que são de mesma área, e essa medida é a mesma dessa daqui (triângulo médio) e essa medida a mesma que essa (triângulo pequeno).

No SG notamos que, de acordo com a posição do segmento $\overline{A B}$ na diagonal do retângulo, era possível visualizar as subfiguras contidas na figura de partida com mais facilidade do que no registro anterior (EG). Duval (2012b) explica que existem fatores internos à figura que disparam ou inibem a visibilidade de operações que modificam a figura, e um deles é o fracionamento da figura em partes elementares.

Nesta atividade observamos que dependendo da ordem de apresentação dos registros figurais, eles podem auxiliar na compreensão do problema, na utilização das hipóteses, bem como na complementaridade de ideias.

Constatamos que os registros na forma de EG e SG foram os que mais propiciaram a resolução correta da atividade, pois esses registros permitiram mais tentativas, já que neles podem ser realizadas operações quantas vezes forem necessárias sem deformar a figura de partida, conforme a descrição do aluno A9 na folha de respostas:

A9: Observando toda a região pude perceber que as áreas de 1 e 2 são iguais, porque os triângulos que estavam ao redor tanto da área 1 quanto os da área 2 são iguais, então as figuras que sobram ou seja as subfiguras 1 e 2 são de mesma área.

Já o MM suscita o uso de recortes e assim limita a opção de refazer a atividade, sem maiores problemas, em busca de extrair conclusões. Além disso, algumas conclusões 
estabelecidas por meio do MM foram incorretas por conta das imperfeições que o material concreto apresenta.

Observamos que muitos alunos mobilizaram outros registros de representação durante as resoluções, principalmente o registro numérico que consistia em fazer o uso de cálculos para verificar empiricamente a igualdade das áreas 1 e 2 . No entanto, tal resolução não pode ser considerada adequada, pois considera casos particulares dos valores para as áreas.

Com o uso do SG, observamos que:

- por meio de suas ferramentas proporcionou ao sujeito movimentar o ponto G (ponto de interseção dos segmentos $\overline{A B}$ e $\overline{J K}$ ), aumentando e diminuindo o tamanho das subfiguras consistindo em modificações óticas, conforme a Figura 4:

Figura 4: Modificações óticas e posicionais no retângulo

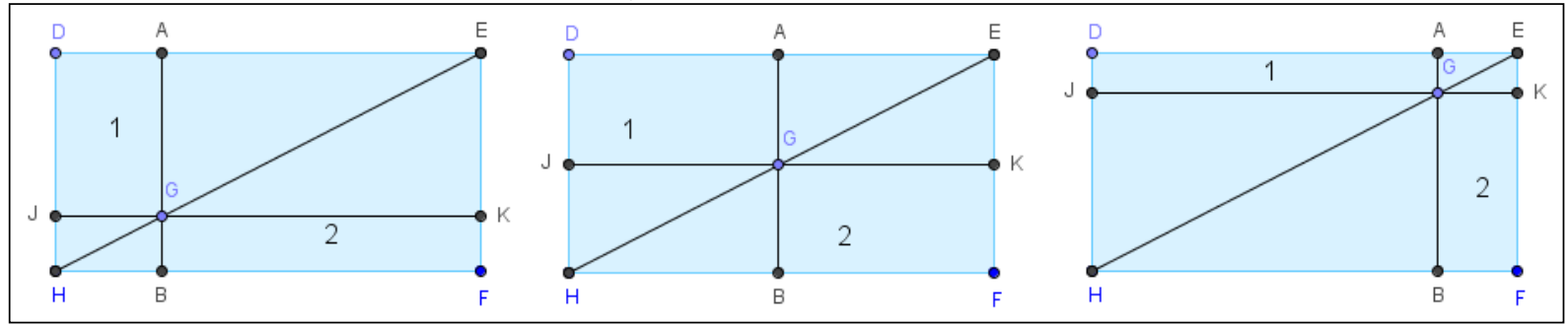

Fonte: Autoras.

- o aluno, ao transladar o segmento $\overline{A B}$ na diagonal do retângulo, realizou modificações posicionais;

- ao movimentar o ponto $\mathrm{G}$ até o ponto médio do segmento $\overline{H E}$ (diagonal) visualizaram-se quatro subfiguras (retângulos) iguais, sendo duas delas as áreas 1 e 2. Desse modo, ocorreram uma operação de reconfiguração e uma exploração heurística da figura, conforme a Figura 5:

Figura 5: Resolução da atividade no registro SG

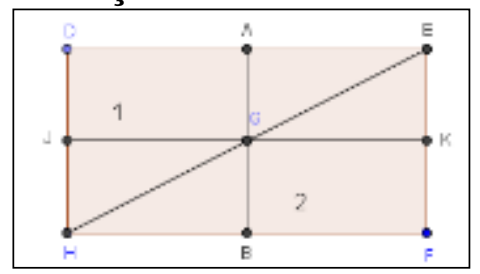

Fonte: Autoras.

- ao movimentar o ponto $\mathrm{G}$ também movimentaram-se os segmentos $\overline{A B}$ e $\overline{J K}$, alterando as áreas das subfiguras; esse fato auxilia na visualização de uma possível modificação mereológica. Desse modo, o software proporcionou congruência entre o registro da língua natural (o enunciado do problema) e o registro figural (representação da figura no software), 
no momento em que o enunciado da atividade diz "qualquer que seja a posição do segmento $\overline{A B} \%$.

Com o uso do MM, foi possível observar que:

- os sujeitos tiveram a ideia de dobrar, recortar, rotacionar, sobrepor e verificar se as áreas eram iguais, realizando modificações mereológicas e posicionais, juntamente a uma exploração heurística mostrando a igualdade das áreas, porém, em uma única posição dos segmentos, conforme a Figura 6:

Figura 6: Resolução da atividade do aluno A9

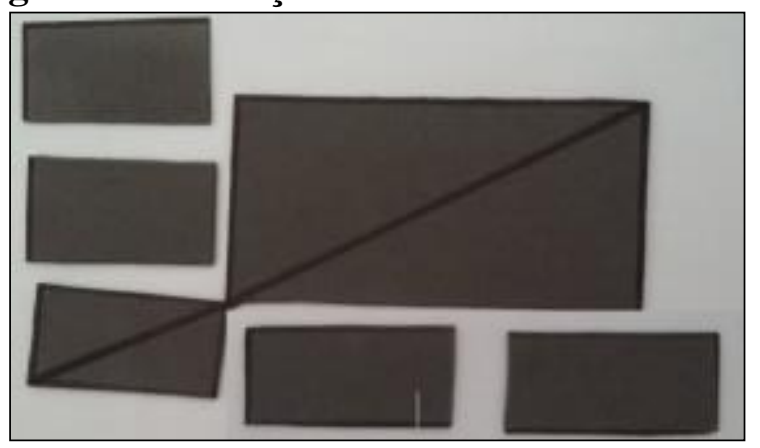

Fonte: Autoras.

- houve a recorrência a recortes da figura inicial na tentativa de comparar as áreas das figuras 1 e 2 ;

- dois alunos realizaram, com o auxílio do lápis, os deslocamentos dos segmentos $\overline{A B}$ e $\overline{J K}$, porém, neste registro, houve pouca congruência entre as hipóteses dadas no enunciado, que afirmava que as áreas 1 e 2 eram iguais em qualquer que fosse a posição do segmento $\overline{A B}$, e os tratamentos figurais realizados, pois somente dois sujeitos simularam o movimento dos segmentos citados, conforme sugeria o enunciado.

Com o uso da EG, foi possível destacar que:

- pela sua característica (figuras construídas manualmente com uso de materiais como lápis, régua, compasso etc.) proporcionou ao sujeito observar mais atentamente as figuras e subfiguras, bem como suas propriedades, a fim de realizar modificações, conforme a Figura 7:

\section{Figura 7: Resolução da atividade do aluno A1}

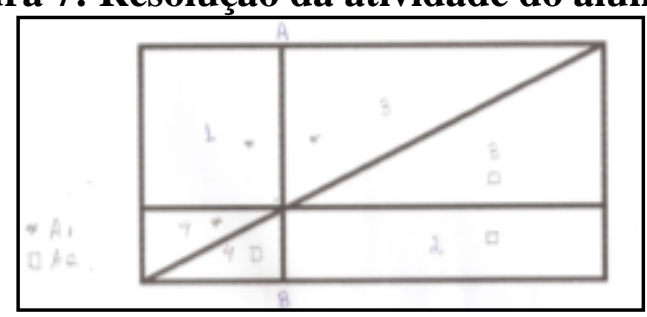

Fonte: Autora. 
- a minoria dos alunos realizou o deslocamento dos segmentos $\overline{A B}$ e $\overline{J K}$, logo, nesse registro, houve pouca congruência entre as hipóteses dadas no enunciado e os tratamentos figurais realizados no que diz respeito a qualquer que seja a posição do segmento $\overline{A B}$.

A modificação mereológica foi realizada nos três registros figurais apresentados e é esta a modificação que proporciona aos alunos a resolução da atividade, embora tenha acontecido, principalmente, no SG e no MM. A modificação ótica e a modificação posicional ocorreram com maior frequência por meio do registro figural representado pelo SG, pelo fato de suas ferramentas possibilitarem de forma dinâmica essas modificações das subfiguras.

Cada registro figural tem aspectos diferentes em conteúdo, ou seja, cada registro tem suas especificidades que levam a diferentes tipos de apreensões operatórias, e em cada tipo de registro figural suscitam-se modificações que resolvem o problema e modificações que se completam a fim de encontrar a solução para o problema. Assim, dependendo do registro apresentado inicialmente os alunos manifestam procedimentos de resoluções diferentes.

Desse modo, a diversidade de registros figurais contribuiu para a visualização de conceitos e propriedades dos objetos geométricos em questão, auxiliando no desenvolvimento do raciocínio, proporcionando diferentes operações e completando um registro figural ao outro.

\section{Referências}

DUVAL, Raymond. Semiosis y pensamento humano: Registros semióticos y Aprendizajes intelectuales. Tradução: Myriam Vega Restrepo. Cali, Colombia: Universidade del Valle, 1999.

Ver e ensinar a matemática de outra forma: entrar no modo matemático de pensar: os registros de representação semióticas. Tânia M. M. Campos (Org.). Tradução: Marlene Alves Dias. São Paulo: PROEM, 2011.

Abordagem cognitiva de problemas de geometria em termos de congruência

(tradução: Méricles Thadeu Moretti). Revemat, Florianópolis, v. 07, n. 1, p. 118-138, 2012a.

Registros de representação semiótica e funcionamento cognitivo do pensamento. (tradução: Méricles Thadeu Moretti). Revemat, Florianópolis, v. 07, n. 2, p. 266-297, 2012 b.

Les conditions cognitives de l'apprentissage de la Géométrie: développement de la visualisation, différenciation des raisonnements et coordination de leur fonctionnements.

Annales de Didactique et de Sciences Cognitive, n. 10, p. 5-53, 2005. 
FLORES, C. R.; MORETTI, M. T. O papel heurístico de uma Figura Geométrica: o caso da operação de Reconfiguração. Anais do VIII Encontro Nacional de Educação Matemática. Universidade de Pernambuco, Recife, 2004.

GÓES, H. C. Expressão Gráfica: Esboço de conceituação. 2012. 123p. Dissertação (Mestrado) - Programa de Pós-Graduação em Educação em Ciências e em Matemática. Universidade Federal do Paraná, Curitiba, 2012.

GRAVINA, M. A.; SANTAROSA, L. M. A aprendizagem da matemática em ambientes informatizados. Anais do IV Congresso RIBIE, 1998.

PASSOS, C. L. B. Materiais manipuláveis como recursos didáticos na formação de professores de matemática. In: LORENZATO, Sérgio (Org.). Laboratório de Ensino de Matemática na formação de professores. Campinas, SP: Autores Associados, 2006. p. 7792. 